\title{
A Novel CDD-OFDM Scheme with Pilot-Aided Channel Estimation
}

\author{
Sili Lu, Student Member, IEEE and Naofal Al-Dhahir, Fellow, IEEE
}

\begin{abstract}
Cyclic delay diversity (CDD) is a low-complexity standard-conformable transmit diversity scheme for coded orthogonal frequency division multiplexing (OFDM) systems. However, it makes channel estimation more challenging due to the increased frequency-selectivity of the equivalent single-input single-ouput channel. In this paper, we propose a novel CDDOFDM scheme with pilot-aided channel estimation for any number of transmit antennas. By alternating and optimizing the cyclic delay parameter over adjacent OFDM symbols, we design a simple yet efficient channel estimation scheme and illustrate its excellent performance for the DVB-T application.
\end{abstract}

Index Terms-OFDM, channel estimation, CDD, SISO, MISO.

\section{INTRODUCTION}

C DD is a low-complexity spatial transmit diversity scheme where multiple antennas transmit cyclicly-shifted versions of the same signal. Essentially, CDD transforms a multiple-input single-output (MISO) frequency-selective channel into an equivalent single-input single-output (SISO) channel with increased frequency-selectivity, where the available spatial diversity is transformed into additional frequency diversity [1]. An OFDM system with forward error correction (FEC) and interleaving can exploit the increased frequency diversity of the equivalent SISO frequency-selective channel to improve the performance. Two other key advantages of CDD-OFDM over other transmit diversity schemes such as orthogonal space-time block codes (STBC) [2][3] are that it is standard-conformable ${ }^{1}$ and it does not incur any additional information rate loss for more than two transmit antennas (unlike orthogonal STBC).

One channel estimation approach for CDD-OFDM is to estimate the SISO-equivalent channel directly [4]. However, in a CDD-OFDM system, the frequency response of the SISO-equivalent channel can vary significantly across adjacent subcarriers due to the increased frequency selectivity. Hence, it becomes more difficult to obtain an accurate interpolation between the pilot subcarriers at practical pilot overhead ratios.

An alternative channel estimation approach for CDDOFDM is based on the MISO model where each channel is estimated individually and then combined to obtain the equivalent estimated SISO channel used for detection [4].

Manuscript received Februry 13, 2008; revised April 29, 2008 and September 8, 2008; accepted November 17, 2008. The associate editor coordinating the review of this letter and approving it for publication was Prof. A. Hjørungnes.

The authors are with University of Texas at Dallas, (e-mail: \{sx1059000, aldhahir\}@utdallas.edu).

This work was supported in part by Semiconductor Research Corporation under contract No. 2005-HJ-1328 and by a gift from Texas Instruments Inc.

Digital Object Identifier 10.1109/TWC.2008.080204.

${ }^{1}$ This implies that the transmission frame format is unchanged and current SISO-OFDM user terminals can decode CDD-OFDM signals.
For a conventional CDD-OFDM system with scattered pilot tones embedded between the data subcarriers in each OFDM symbol, as we show in Section III, the CDD-encoded pilot codeword matrix is singular. Even when a Bayesian channel estimator is used at the pilot locations, it results in an irreducible channel estimation mean square error as SNR is increased. These considerations motivated us to design a new CDD-OFDM transmission scheme where the pilot codewords can be made orthogonal to achieve the Cramer-Rao bound on the channel estimation error variance. Our simulation results show that MISO channel estimation for our proposed CDDOFDM scheme achieves significant performance improvement over SISO-equivalent and MISO channel estimation schemes for conventional CDD-OFDM at the same pilot overhead ratio.

The rest of this paper is organized as follows. The system model is described in Section II and the existing methods for CDD-OFDM channel estimation are reviewed in Section III. We propose our new CDD-OFDM scheme and its associated channel estimation algorithm for two transmit antennas in Section IV and generalize it to an arbitrary number of transmit antennas in Section V. Simulation results are presented in Section VI, and conclusions are drawn in Section VII.

Notation: We use $(\cdot)^{T}$ to denote the transpose, $(\cdot)^{H}$ the complex-conjugate transpose, and $(\cdot)_{N}$ the modulo- $N$ operation. The estimated value of a variable $a$ is denoted by $\hat{a}$. $\mathbf{I}_{N}$ denotes the $N \times N$ identity matrix and $\operatorname{Diag}(\mathbf{v})$ denotes a diagonal matrix with diagonal elements given by the vector v.

\section{SYSTEM MODEL}

For a conventional CDD-OFDM system with $N_{T}$ transmit antennas, ${ }^{2}$ each antenna introduces a different cyclic delay $\Delta_{n}, n=1, \ldots, N_{T}$, resulting in the following transmitted time-domain signal from the $n$-th antenna

$$
x_{t}^{(n)}=x_{\left(t-\Delta_{n}\right)_{N}} ; \quad 0 \leq t \leq N-1 ; \quad 1 \leq n \leq N_{T}
$$

where $N$ is the inverse discrete Fourier transform (IDFT) size. A cyclic delay by $\Delta_{n}$ samples in the time-domain corresponds to the following phase shift at the $i$-th subcarrier

$$
\varphi_{i}^{(n)}=2 \pi i \Delta_{n} / N
$$

Hence, the $i$-th frequency-domain subcarrier $X_{i}^{(n)}$ is related to the time-domain signal $x_{t}^{(n)}$ in (1) by the relation

$$
\begin{aligned}
x_{t}^{(n)} & =\frac{1}{N} \sum_{i=0}^{N-1} X_{i}^{(n)} e^{j 2 \pi t i / N} \\
& =\frac{1}{N} \sum_{i=0}^{N-1} X_{i} e^{-j \varphi_{i}^{(n)}} e^{j 2 \pi t i / N}
\end{aligned}
$$

\footnotetext{
${ }^{2}$ Throughout this paper, we assume one receive antenna. The extension to multiple receive antennas is straightforward.
} 
Generally, the frequency-domain input-output relation for a MISO-OFDM system at the $i$-th subcarrier is $Y_{i}=$ $\sum_{n=1}^{N_{T}} X_{i}^{(n)} G_{i}^{(n)}+V_{i}$ where $Y_{i}, G_{i}$ and $V_{i}$ are the received signal, the channel frequency response, and the noise (assumed white Gaussian with variance $\sigma_{v}^{2}$ ) at the $i$-th subcarrier, respectively. Using (3), the received signal at the $i$-th subcarrier can be expressed as

$$
\begin{aligned}
Y_{i} & =X_{i}\left(\sum_{n=1}^{N_{T}} G_{i}^{(n)} e^{-j \varphi_{i}^{(n)}}\right)+V_{i} \\
& =X_{i} G_{i}^{\text {eqv }}+V_{i} ; \quad 0 \leq i \leq N-1
\end{aligned}
$$

where we define $G_{i}^{\text {eqv }} \stackrel{\text { def }}{=} \sum_{n=1}^{N_{T}} G_{i}^{(n)} e^{-j \varphi_{i}^{(n)}}$ to be the SISOequivalent channel frequency response (CFR) at the $i$-th subcarrier. Corresponding to this equivalent CFR, the following equivalent channel impulse response (CIR) can be defined, which describes the time-domain SISO-equivalent channel of the CDD-OFDM system [4]

$$
h^{\mathrm{eqv}}(\tau)=\sum_{n=1}^{N_{T}} \sum_{q=0}^{\nu} h_{q}^{(n)} \cdot \delta\left(\tau-\tau_{q}^{(n)}-\Delta_{n} T_{\mathrm{spl}}\right)
$$

where $h_{q}^{(n)}$ and $\tau_{q}^{(n)}$ are the gain and time delay for the $q$-th tap of the CIR from the $n$-th transmit antenna, respectively, and $\nu$ is the channel memory normalized to the OFDM sampling period $T_{\text {spl }}$. It is clear from (5) that all CIRs from the different transmit antennas are concatenated together at different delays, making the SISO-equivalent CIR of the CDD system much longer than the CIR of the individual channels, resulting in more severe channel frequency selectivity. It is also important to note that there will be no additional intersymbol interference (ISI) even if the channel memory of the SISO-equivalent CIR is larger than the cyclic prefix $(\mathrm{CP})$, since the CDD operations are done before the $\mathrm{CP}$ insertion (see [5] and Fig. 1).

\section{COnVEntional CDD-OFDM Channel Estimation}

Considering a scattered pilot structure, we assume that in each OFDM symbol there are $P$ equally-spaced unitmagnitude pilots at subcarriers $p(0), \ldots, p(P-1)$. Then, using the equivalent model in (4), we calculate the SISO-equivalent $\mathrm{CFR}$ at the pilot subcarrier locations as follows

$$
\hat{G}_{p(i)}^{\mathrm{eqv}}=\frac{Y_{p(i)}}{X_{p(i)}} ; \quad 0 \leq i \leq P-1
$$

Subsequently, these estimates are interpolated to obtain an estimate of the entire equivalent CFR at all subcarriers. However, since the SISO-equivalent channel is highly frequency-selective (due to CDD effects), its CFR can exhibit significant fluctuations across adjacent subcarriers, making it very difficult to obtain a reliable interpolation between pilot locations at practical pilot overhead levels.

An alternative channel estimation approach is based on the original MISO channel model where each CFR coefficient $\hat{G}_{i}^{(n)}$ is estimated individually. Assume that we have two transmit antennas, with the first antenna transmitting the original version of the signal, i.e. $\Delta_{1}=0$ and the second antenna transmitting a cyclicly-shifted version of the signal by $\Delta_{2} \neq 0$ samples. Denoting the transmitted and received $k$-th OFDM symbols at the $i$-th subcarrier by $X_{i}^{k}$ and $Y_{i}^{k}$, respectively, and using (2), we can express the input-output relation for OFDM symbols $k$ and $(k+1)$ as follows

$$
\begin{aligned}
Y_{i}^{k}= & X_{i}^{k}\left(G_{i}^{(1), k}+G_{i}^{(2), k} e^{-j 2 \pi \cdot i \Delta_{2} / N}\right)+V_{i}^{k} \\
Y_{i}^{k+1}= & X_{i}^{k+1}\left(G_{i}^{(1), k+1}+G_{i}^{(2), k+1} e^{-j 2 \pi \cdot i \Delta_{2} / N}\right) \\
& +V_{i}^{k+1}
\end{aligned}
$$

Assuming the channel remains fixed over two adjacent OFDM symbols, i.e. the channel coherence time is much larger than the OFDM symbol duration ${ }^{3}$, we have $G_{i}^{(n), k} \approx$ $G_{i}^{(n), k+1} \stackrel{\text { def }}{=} G_{i}^{(n)}$ for $n=1,2$. Under this assumption, (7) can be written as follows

$$
\begin{aligned}
{\left[\begin{array}{c}
Y_{i}^{k} \\
Y_{i}^{k+1}
\end{array}\right]=} & {\left[\begin{array}{cc}
X_{i}^{k} & X_{i}^{k} e^{-j 2 \pi \cdot i \Delta_{2} / N} \\
X_{i}^{k+1} & X_{i}^{k+1} e^{-j 2 \pi \cdot i \Delta_{2} / N}
\end{array}\right]\left[\begin{array}{c}
G_{i}^{(1)} \\
G_{i}^{(2)}
\end{array}\right] } \\
& +\left[\begin{array}{c}
V_{i}^{k} \\
V_{i}^{k+1}
\end{array}\right]
\end{aligned}
$$

or in compact matrix form as

$$
\mathbf{Y}_{i}=\mathbf{X}_{i} \mathbf{G}_{i}+\mathbf{V}_{i}
$$

It can be readily checked that $\mathbf{X}_{i}$ is a singular matrix; hence, even the classical least-squares (LS) estimator is not defined in this case. However, if the covariances matrices of $\mathbf{G}_{i}$ and $\mathbf{V}_{i}$ are known, we can calculate the Bayesian minimum mean square error (BMMSE) estimator of $\mathbf{G}_{i}$ given by [6] ${ }^{4}$

$$
\begin{aligned}
\hat{\mathbf{G}}_{i, \mathrm{BMMSE}} & =\mathbf{R}_{G} \mathbf{X}_{i}^{H}\left(\mathbf{X}_{i} \mathbf{R}_{G} \mathbf{X}_{i}^{H}+\mathbf{R}_{V}\right)^{-1} \mathbf{Y}_{i} \\
& =\mathbf{X}_{i}^{H}\left(\mathbf{X}_{i} \mathbf{X}_{i}^{H}+\sigma_{v}^{2} \mathbf{I}_{2}\right)^{-1} \mathbf{Y}_{i}
\end{aligned}
$$

where $\mathbf{R}_{V}=\sigma_{v}^{2} \mathbf{I}_{2}$ is the noise covariance matrix and $\mathbf{R}_{G}=\mathbf{I}_{2}$ is the covariance matrix for the unknown $\mathbf{G}_{i}$. Since the channels from the different transmit antennas are assumed to be uncorrelated (i.e. $E\left[h_{i}^{(1)}\left(h_{j}^{(2)}\right)^{*}\right]=0$ in (5)), it is easy to show $E\left[G_{i}^{(1)}\left(G_{i}^{(2)}\right)^{*}\right]=0$. If we further assume $\Sigma_{q=0}^{\nu} E\left[\left|h_{q}^{(i)}\right|^{2}\right]=1$, we can also show that $E\left[G_{i}^{(i)}\left(G_{i}^{(i)}\right)^{*}\right]=$ 1 ; hence, $\mathbf{R}_{G}=\mathbf{I}_{2}$. The error covariance matrix in estimating $\mathbf{G}_{i}$ is given by [6]

$$
\begin{aligned}
\mathbf{C}_{e} & =\left(\mathbf{R}_{G}^{-1}+\mathbf{X}_{i}^{H} \mathbf{R}_{V}^{-1} \mathbf{X}_{i}\right)^{-1} \\
& =\left(\mathbf{I}_{2}+\gamma\left[\begin{array}{cc}
1 & e^{-j 2 \pi \cdot i \Delta_{2} / N} \\
e^{j 2 \pi \cdot i \Delta_{2} / N} & 1
\end{array}\right]\right)^{-1} \\
& =\frac{1}{2 \gamma+1}\left[\begin{array}{cc}
1+\gamma & -\gamma e^{-j 2 \pi \cdot i \Delta_{2} / N} \\
-\gamma e^{j 2 \pi \cdot i \Delta_{2} / N} & 1+\gamma
\end{array}\right]
\end{aligned}
$$

where we denote the normalized SNR as $\gamma=\frac{\left|X_{i}^{k}\right|^{2}+\left|X_{i}^{k+1}\right|^{2}}{\sigma_{v}^{2}}=$ $\frac{2 E_{s}}{\sigma_{v}^{2}}$, and $E_{s}=\left|X_{i}^{k}\right|^{2}$ is the pilot subcarrier energy. Hence, the MSE for $\hat{G}_{i}^{(m)}$ is given by

$$
\operatorname{MSE}\left(\hat{G}_{i}^{(m)}\right)=\left[\mathbf{C}_{e}\right]_{m, m}=\frac{\gamma+1}{2 \gamma+1} ; \quad m=1,2
$$

Clearly, the MSE of this channel estimator is lower bounded by $1 / 2$ as SNR goes to infinity which is not satisfying. This motivates our design of a new CDD-OFDM scheme which ensures orthogonality of the pilot codewords ${ }^{5}$.

\footnotetext{
${ }^{3}$ We will verify the validity of this assumption for the DVB $2 \mathrm{~K}$ mode system, even at high mobile speeds, in Section VI.

${ }^{4}$ The key difference between the classical and Bayesian estimation approaches is that in the former approach the unknown is assumed deterministic while in the latter approach it is assumed random [6].

${ }^{5}$ Orthogonality of the codewords not only ensures invertibility but also minimizes the total estimation error variance.
} 


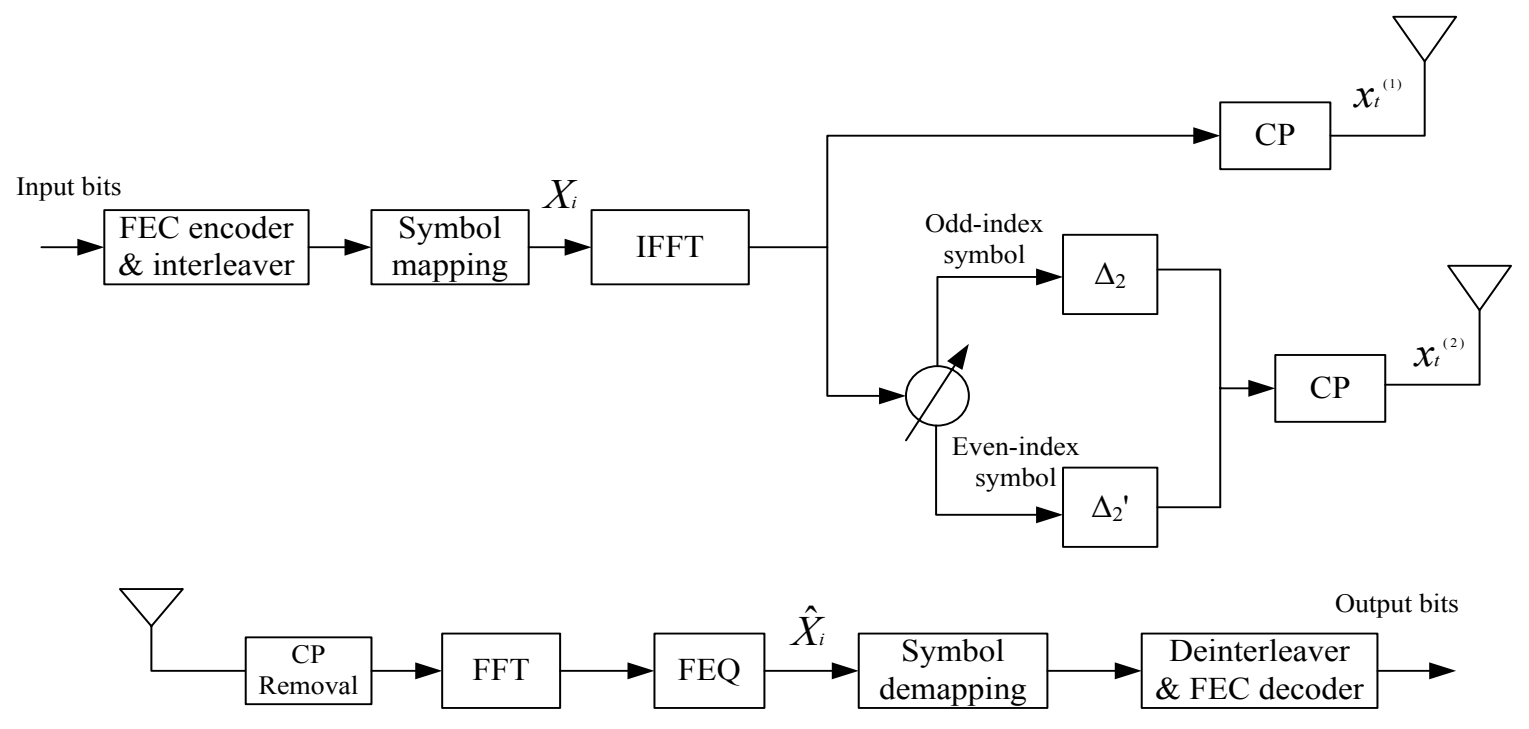

Fig. 1. Proposed CDD-OFDM scheme for 2 transmit antennas. CP denotes the cyclic prefix

\section{PROPOSED CDD-OFDM SCHEME}

To illustrate the main idea, we start by assuming two transmit antennas, with the first antenna transmitting the original version of the signal, i.e. $\Delta_{1}=0$. For the second transmit antenna, we use alternating cyclic delay parameters of $\Delta_{2}$ and $\Delta_{2}^{\prime}$, as illustrated in Fig. 1. In other words, for the $k$ th OFDM symbol, we cyclicly shift the time-domain OFDM symbol by $\Delta_{2}$ samples while for the $(k+1)$ symbol we shift it by $\Delta_{2}^{\prime} \neq \Delta_{2}$ samples. In this case, the pilot codeword $\mathbf{X}_{i}$ in (8) will be replaced by

$$
\mathbf{X}_{i}=\left[\begin{array}{cc}
X_{i}^{k} & X_{i}^{k} e^{-j 2 \pi \cdot i \Delta_{2} / N} \\
X_{i}^{k+1} & X_{i}^{k+1} e^{-j 2 \pi \cdot i \Delta_{2}^{\prime} / N}
\end{array}\right]
$$

Then, we use the fact (see Theorem 4.1 and Example 4.3 in [6]) that the optimal choice for $\mathbf{X}_{i}$ is to make it orthogonal at pilot locations, i.e. $\mathbf{X}_{i}^{H} \mathbf{X}_{i}=2 E_{s} \mathbf{I}_{2}$. If this can be achieved, the efficient estimator (i.e. the one which achieves the CramerRao bound) is given by [6]

$$
\hat{\mathbf{G}}_{i}=\left(\mathbf{X}_{i}^{H} \mathbf{R}_{V}^{-1} \mathbf{X}_{i}\right)^{-1} \mathbf{X}_{i}^{H} \mathbf{R}_{V}^{-1} \mathbf{Y}_{i}=\frac{1}{2 E_{s}} \mathbf{X}_{i}^{H} \mathbf{Y}_{i}
$$

The resulting covariance matrix for $\hat{\mathbf{G}}_{i}$ is $\mathbf{C}_{e}=$ $\left(\mathbf{X}_{i}^{H} \mathbf{R}_{V}^{-1} \mathbf{X}_{i}\right)^{-1}=\frac{1}{\gamma} \mathbf{I}_{2}$ and the variance of $\hat{G}_{i}^{(m)}$ is $1 / \gamma$. For a fair comparison with the BMMSE estimator for the conventional CDD-OFDM system, we can also implement a Bayesian MMSE channel estimator for our proposed CDDOFDM and further reduce the channel estimation MSE to $\frac{1}{\gamma+1}$ (see Fig. 2). However, this Bayesian estimator is biased and requires knowledge of the second-order statistics of the noise and frequency-domain channel coefficients. A detailed performance comparison between the different estimators is presented in Section VI.

Next, we show how to design our proposed CDD-OFDM scheme to ensure the orthogonality of $\mathbf{X}_{i}$ at pilot locations, i.e. $\mathbf{X}_{p(i)}^{H} \mathbf{X}_{p(i)}=2 E_{s} \mathbf{I}_{2}$ where $p(i)$ is a pilot index. From (11), this orthogonality condition implies that

$$
E_{s} e^{-j 2 \pi p(i) \Delta_{2} / N}+E_{s} e^{-j 2 \pi p(i) \Delta_{2}^{\prime} / N}=0
$$

In order for (13) to hold, we must have

$$
2 \pi \cdot p(i)\left(\Delta_{2}^{\prime}-\Delta_{2}\right) / N=\pi \pm 2 l \pi \text { for } l=0,1, \ldots
$$

Assuming an equal pilot spacing of $d$ subcarriers, we can write the $i$-th pilot index as $p(i)=\delta_{0}+i d$ where $\delta_{0}$ is the subcarrier index for the first pilot. Substituting into (14), we have

$$
2\left(\delta_{0}+i d\right) \frac{\Delta_{2}^{\prime}-\Delta_{2}}{N}=1 \pm 2 l
$$

For a given $N$ and $\delta_{0}$, to satisfy (15), we set

$$
\Delta_{2}^{\prime}-\Delta_{2}=\frac{N}{2 \delta_{0}}
$$

Hence, (15) becomes

$$
i \frac{d}{\delta_{0}}= \pm 2 l
$$

Since the right-hand side of (17) is always an even integer, we can ensure that (17) is satisfied at all pilot subcarrier locations by selecting the ratio $\frac{d}{\delta_{0}}$ to be an even integer. Note that (16) only specifies the cyclic delays difference over the 2 adjacent OFDM symbols for the second transmit antenna. For frequency-selective channels, the condition $\Delta_{2} \geq \Delta_{1}+\nu$ must be satisfied to exploit the available multipath diversity gain [1]. Since $\Delta_{1}=0$, we can set $\Delta_{2}$ to be any integer larger than or equal to $\nu$, and set $\Delta_{2}^{\prime}$ to satisfy (16). From (17), we set $\delta_{0}$ to be

$$
\delta_{0}=\frac{d}{2 r}
$$

where $r$ can be any integer ranging from 1 to $d / 2$. This implies that

$$
\left(\Delta_{2}^{\prime}-\Delta_{2}\right)=r N / d
$$

In practice, $d$ must be selected to fulfill the following sampling theorem requirement in the frequency-domain

$$
d \leq \frac{1}{K \tau_{\max } \Delta_{f}}
$$


where $\tau_{\max }=\nu T_{\mathrm{spl}}$ is the CIR maximum delay spread, $\Delta_{f}$ is the subcarrier spacing and the constant $K$ corresponds to an oversampling rate which is typically used to suppress noise and improve the channel estimation performance. Since our channel estimation scheme estimates each channel individually based on the MISO model, $\tau_{\max }$ in this case equals to the delay spread of each individual channel.

\section{Extension to the $N_{T}>2$ CAse}

In this section, we extend our proposed CDD-OFDM scheme and its channel estimation pilot design to the $N_{T}>2$ case, for which the pilot matrix in (11) generalizes to

$$
\begin{aligned}
& \mathbf{X}_{p(i)}= \\
& {\left[\begin{array}{cclc}
X_{p(i)}^{k} & X_{p(i)}^{k} e\left(\Delta_{2}^{1}\right) & \cdots & X_{p(i)}^{k} e\left(\Delta_{N_{T}}^{1}\right) \\
X_{p(i)}^{k+1} & X_{p(i)}^{k+1} e\left(\Delta_{2}^{2}\right) & \cdots & X_{p(i)}^{k+1} e\left(\Delta_{N_{T}}^{2}\right) \\
\vdots & \vdots & \ddots & \vdots \\
X_{p(i)}^{k+N_{T}-1} & X_{p(i)}^{k+N_{T}-1} e\left(\Delta_{2}^{N_{T}}\right) & \cdots & X_{p(i)}^{k+N_{T}-1} e\left(\Delta_{N_{T}}^{N_{T}}\right)
\end{array}\right]}
\end{aligned}
$$

where $\Delta_{n}^{m}$ denotes the cyclic delay parameter for the $n$-th transmit antenna at the $m$-th OFDM symbol and we define $e(x) \stackrel{\text { def }}{=} \exp (-j 2 \pi p(i) x / N)$. It is not hard to show that $\mathbf{X}_{p(i)}$ can be factorized as $\mathbf{X}_{p(i)}=$ $\mathbf{U X}_{p(i)}^{\prime} \mathbf{W}$, where $\mathbf{U}$ and $\mathbf{W}$ are diagonal matrices given by $\mathbf{U}=\operatorname{Diag}\left(\left[X_{p(i)}^{k}, \ldots, X_{p(i)}^{k+N_{T}-1}\right]\right), \quad \mathbf{W}=$ $\operatorname{Diag}\left(\left[1, e\left(\Delta_{2}^{1}\right), \ldots, e\left(\Delta_{N_{T}}^{1}\right)\right]\right)$ and $\mathbf{X}_{p(i)}^{\prime}$ is given by

$$
\mathbf{X}_{p(i)}^{\prime}=\left[\begin{array}{cccc}
1 & 1 & \cdots & 1 \\
1 & e\left(\Delta_{2}^{2}-\Delta_{2}^{1}\right) & \cdots & e\left(\Delta_{N_{T}}^{2}-\Delta_{N_{T}}^{1}\right) \\
\vdots & \vdots & \ddots & \vdots \\
1 & e\left(\Delta_{2}^{N_{T}}-\Delta_{2}^{1}\right) & \cdots & e\left(\Delta_{N_{T}}^{N_{T}}-\Delta_{N_{T}}^{1}\right)
\end{array}\right]
$$

Since $\mathbf{U}$ and $\mathbf{W}$ are diagonal matrices, we have $\mathbf{U} \mathbf{U}^{H}=$ $E_{s} \mathbf{I}_{N_{T}}$ and $\mathbf{W} \mathbf{W}^{H}=\mathbf{I}_{N_{T}}$. Hence, to make $\mathbf{X}_{p(i)}$ orthogonal, it suffices to make $\mathbf{X}_{p(i)}^{\prime}$ orthogonal since $\mathbf{X}_{p(i)}^{H} \mathbf{X}_{p(i)}=$ $E_{s} \mathbf{W}^{H} \mathbf{X}_{p(i)}^{\prime H} \mathbf{X}_{p(i)}^{\prime} \mathbf{W}$. The special structure of $\mathbf{X}_{p(i)}^{\prime}$ in (22) suggests the following choice for $\Delta_{n}^{m}$ to make $\mathbf{X}_{p(i)}^{\prime}$ an DFT matrix and hence orthogonal.

$$
\Delta_{n}^{m}-\Delta_{n}^{1}=\frac{(m-1)(n-1) N}{N_{T} \delta_{0}}
$$

where $2 \leq n \leq N_{T}$ and $1 \leq m \leq N_{T}$. Substituting for $p(i)$ by $\delta_{0}+i d$ and using (23), the $(m, n)$ element of $\mathbf{X}_{p(i)}^{\prime}$ in (22) can be expressed as

$$
\begin{aligned}
X_{p(i)}^{\prime}(m, n)= & \exp \left(\frac{-j 2 \pi p(i)\left(\Delta_{n}^{m}-\Delta_{n}^{1}\right)}{N}\right) \\
= & \exp \left(\frac{-j 2 \pi(n-1)(m-1)}{N_{T}}\right) \times \\
& \exp \left(\frac{-j 2 \pi i d(n-1)(m-1)}{N_{T} \delta_{0}}\right)
\end{aligned}
$$

We make the second term on the right hand side of (24) equal to one by setting

$$
(d)_{N_{T} \delta_{0}}=0
$$

In other words, $d$ is set to be an integer multiple of $N_{T} \delta_{0}$. Then, $X_{p(i)}^{\prime}(m, n)=\exp \left(\frac{-j 2 \pi(n-1)(m-1)}{N_{T}}\right)$ becomes equal to the $(m, n)$ element of the DFT matrix of size $N_{T}$. Hence, both $\mathbf{X}_{p(i)}^{\prime}$ and $\mathbf{X}_{p(i)}$ become orthogonal matrices as desired to minimize the channel estimation error variance. We also note from (25) that the maximum number of transmit antennas which can be supported by our proposed CDD-OFDM scheme is equal to $d$. Similar to the $N_{T}=2$ case, we set $\delta_{0}$ according to $(25)$ as

$$
\delta_{0}=\frac{d}{r N_{T}}
$$

where $r$ is any integer ranging from 1 to $d / N_{T}$. Hence, (23) becomes

$$
\Delta_{n}^{m}-\Delta_{n}^{1}=\frac{r(m-1)(n-1) N}{d} ;
$$

where $2 \leq n \leq N_{T}$ and $1 \leq m \leq N_{T}$.

To summarize, the pilot design procedure for a system with given $N, N_{T}$ and $d$ starts by choosing the pilot offset parameter $\delta_{0}$ that satisfies (26) and then sets the cyclic delay parameters according to (27). It can be easily verified that (18) and (19) are special cases of (26) and (27), respectively, when $N_{T}=2$. We also note that our design procedure only specifies the cyclic delays difference for each antenna. Hence, we have the freedom to choose the delay parameters for the first OFDM symbol, as long as it is larger than the channel memory, i.e. $\Delta_{n}^{1}>\nu$ for $2 \leq n \leq N_{T}$ [1].

The main assumption in our proposed CDD-OFDM scheme is that the channel remains constant over $N_{T}$ consecutive OFDM symbols. This requires the channel coherence time, which is inversely proportional to the Doppler frequency, to be much larger than the time duration of $N_{T}$ OFDM symbols. A popular rule of thumb to calculate the channel coherence time is $T_{c}=\frac{0.423}{f_{d}}$ [7]. Here, $f_{d}$ is the Doppler frequency defined by $f_{d}=\frac{v}{c} f_{c}$ where $v, c$ and $f_{c}$ are the vehicle speed, the speed of light and the carrier frequency, respectively. If we set the coherence time to be 10 times larger than $N_{T}$ OFDM symbol durations (each denoted by $T_{s}$ ), the vehicle speed must satisfy the condition

$$
v<\frac{0.0423 c}{N_{T} T_{s} f_{c}}
$$

\section{Simulation Results}

We evaluated the performance of our proposed pilot-aided CDD-OFDM scheme by simulations for the Digital Video Broadcasting-Terrestrial (DVB-T) $2 \mathrm{~K}$ mode system with $N=$ 2048 subcarriers[8] ${ }^{6}$. We assumed the typical urban TU06 channel delay profile defined by the COST 207 project with 6 taps in the continuous-time domain, $8 \mathrm{MHz}$ channel bandwidth, and a carrier frequency of $600 \mathrm{MHz}$ (typical UHF channel for terrestrial broadcasting). A 64-state rate-1/2 convolutional code concatenated with an outer Reed-Solomon (RS) code [8] and a matrix interleaver of block length 256 were implemented. The coded bits were block interleaved and mapped into QPSK symbols.

We first compare the MSE performance for MISO channel estimation using the conventional CDD-OFDM and our proposed CDD-OFDM scheme. The MSE is based on the estimated channel coefficients $\hat{G}_{i}^{(n)}$ for the individual MISO

\footnotetext{
${ }^{6}$ Some modifications to the existing DVB-T pilot structure have to be made to apply our proposed CDD-OFDM scheme.
} 


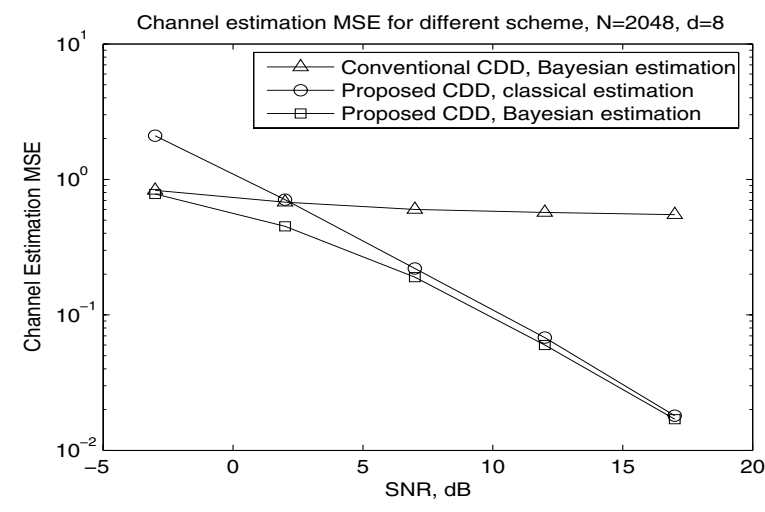

Fig. 2. MSE performance of 2-TX MISO channel estimation for the conventional and proposed CDD-OFDM schemes

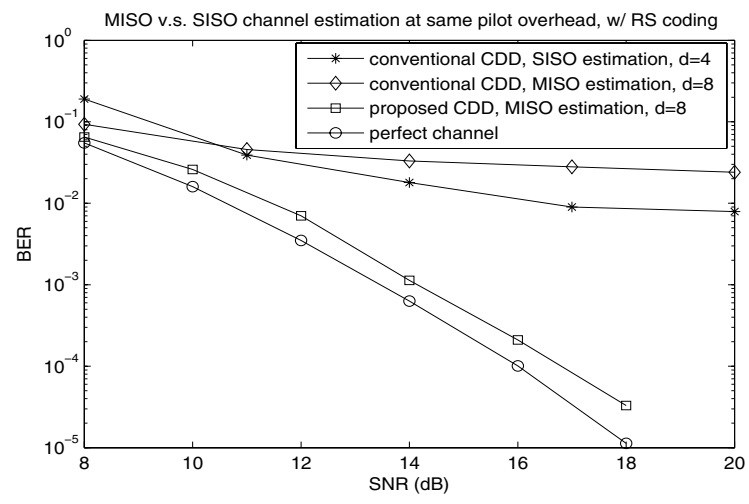

Fig. 3. BER comparison between different channel estimation schemes at the same pilot overhead ratio

channels at the pilot subcarrier locations before interpolation. It can be seen from Fig. 2 that the MSE of the Bayesian MMSE estimator for the conventional CDD-OFDM scheme has a floor of $1 / 2$ which is consistent with our MSE analysis in Section III. By making the pilot codewords orthogonal, our proposed CDD-OFDM scheme eliminates the MSE floor using the efficient classical estimator in (12), as shown in Fig. 2. By equating the MSE equations for the two estimators (i.e. setting $\frac{\gamma+1}{2 \gamma+1}=\frac{1}{\gamma}$ ), we find that the crossing point is at $\gamma \approx 2 d B$, as verified in Fig. 2. To further improve the channel estimator performance for our proposed CDD-OFDM scheme at low SNR, we can also implement the Bayesian MMSE channel estimator, but it requires knowledge of the second order statistics of noise and frequency-domain channel coefficients. It can also be observed from Fig. 2 that the efficient classical and BMMSE estimators of our proposed CDD-OFDM scheme achieve comparable MSE performance at medium-to-high SNR. Hence, we implement the efficient classical estimator for our proposed CDD-OFDM scheme in the subsequent simulations since it does not require knowledge of the second order statistics.

Next, we compare the BER performance for our proposed CDD-OFDM scheme with the conventional CDDOFDM scheme. For MISO channel estimation of our proposed scheme, we choose a pilot spacing of $d=8$ and $\operatorname{set}^{7} r=1$ and $\delta_{0}=4$ according to (18) which, using (19), results in $\Delta_{2}^{\prime}-\Delta_{2}=N / 8=256$. The cyclic delay parameters we implemented to achieve the best performance were $\Delta_{2}=64$ and $\Delta_{2}^{\prime}=320$ for odd and even-indexed OFDM symbols, respectively, where $\Delta_{2}$ is set equal to the channel memory ${ }^{8}$. This design will make $\mathbf{X}_{p(i)}$ orthogonal and we can estimate the two channels at the pilot locations according to (12). Using $\hat{G}_{p(i)}^{(n)}$, we linearly interpolate and estimate the entire frequency response for each channel separately, and finally we combine the two estimated channels to get the SISO-equivalent CFR at subcarrier $i$ as $\hat{G}_{i}^{\text {eqv }}=\hat{G}_{i}^{(1)}+\hat{G}_{i}^{(2)} e^{-2 \pi \cdot i \Delta / N}$ where $\Delta$ alternates between $\Delta_{2}$ and $\Delta_{2}^{\prime}$. Finally, this channel estimate is used to compute the coefficients of the 1-tap frequencydomain equalizer (FEQ) for each subcarrier. As a comparison benchmark, we also investigate the performances for SISOequivalent and MISO channel estimation for the conventional CDD-OFDM system where the same cyclic delay parameter $\Delta_{2}=192$ is used. Unlike the MISO approach, the SISOequivalent channel estimation approach estimates each OFDM symbol individually, while for the MISO approach, $N_{t}$ OFDM symbols are grouped together to form one pilot codeword (see (21)). Hence, for the SISO-equivalent estimation approach, we insert one pilot tone for every four subcarriers $(d=4)$ and perform channel estimation every other OFDM symbol, so that the overall pilot overhead is the same as the MISO channel estimation approach (with $d=8$ and pilot tones in each OFDM symbol) to ensure a fair comparison. For the MISO channel estimation approach for conventional CDD-OFDM, we implement the Bayesian estimator in (9). Fig. 3 compares the BER performance of these three approaches with the case of perfect channel knowledge. It can be seen that our proposed MISO channel estimation approach significantly outperforms the conventional CDD-OFDM scheme with SISO-equivalent channel estimation, since the SISO-equivalent CFR exhibits significant variations and interpolation becomes unreliable even with more densely-spaced pilots (corresponding to $d=$ 4). The MISO channel approach for conventional CDD also has an error floor due to the large channel estimation error variance caused by the singularity of the pilot codeword matrices.

Next, we evaluate the BER performance of our proposed CDD-OFDM scheme with $N_{T}=2$ and different pilot spacings $d$. The TU-06 channel profile has a maximum delay of $\tau_{\max }=5 \mu \mathrm{s}$ and the subcarrier spacing for the $2 \mathrm{~K}$ mode $8 \mathrm{MHz}$ channel is $\Delta_{f}=4 \mathrm{KHz}$; hence, the pilot spacing must be $d<25$ according to (20) assuming an oversampling rate of $K=2$. To ensure that the cyclic delay parameter difference in (19) is an integer, we consider $d=2,4,8$ and 16 and design $\delta_{0}$ and $\left(\Delta_{2}-\Delta_{2}^{\prime}\right)$ according to (18) and (19), respectively. Fig. 4 depicts the BER performance for different pilot spacings. Since the pilot overhead ratio changes with $d$, so does the effective information signal energy level. We account for this SNR loss due to pilot overhead in calculating the SNR level on the X-axis of Fig. 4. It can be seen from

\footnotetext{
${ }^{7}$ Although not shown here due to space limitation, we found that setting $r=1$ results in the best BER performance.

${ }^{8} \mathrm{We}$ verified that, in agreement with [9], setting $\Delta_{2}$ less than the channel memory results in significant performance degradation.
} 


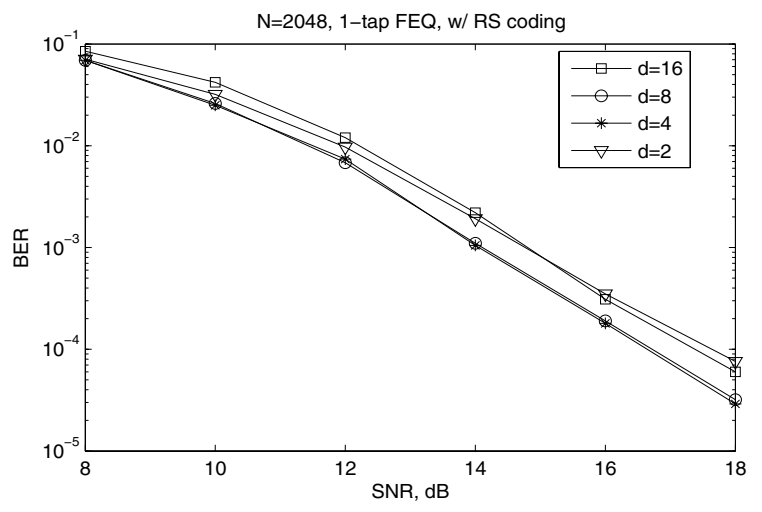

Fig. 4. BER performance of proposed 2-TX CDD-OFDM at different pilot spacing with estimated channel information

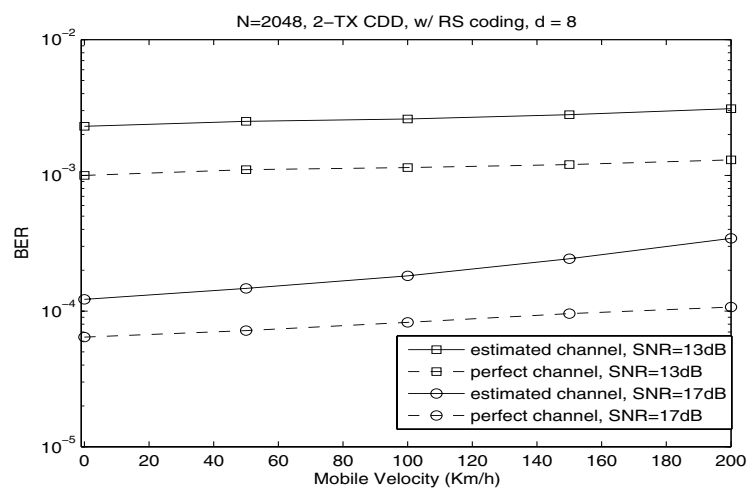

Fig. 5. BER performance of proposed 2-TX CDD-OFDM v.s. mobile velocity

Fig. 4 that $d=8$ achieves the best information rate and BER performance trade-off. Choosing a larger $d$ degrades the BER performance due to degradation in channel estimation quality while choosing a smaller $d$ reduces the effective information rate due to increased pilot overhead.

We also investigate the robustness of our proposed CDDOFDM scheme and its channel estimation pilot design to mobility by evaluating the BER performance at different vehicle speeds. At a fixed SNR of $17 \mathrm{~dB}$, Fig. 5 shows that there is negligible performance degradation due to mobility for speeds up to $100 \mathrm{Km} / \mathrm{h}$. At $13 \mathrm{~dB}$ SNR where intercarrier interference effects due to mobility become less pronounced, our scheme can support even higher speeds up to $150 \mathrm{Km} / \mathrm{h}$ with negligible performance loss from the case of no mobility. Since the DVB symbol duration for the $2 \mathrm{~K}$ mode is approximately $250 \mu s$ [8], for a two-antenna system $\left(N_{T}=2\right)$ operating at carrier frequency of $600 \mathrm{MHz}$, we can assume a fixed channel over 2 OFDM symbols for mobile speeds up to $150 \mathrm{~km} / \mathrm{h}$ according to (28) which is in agreement with Fig. 5.

Finally, Fig. 6 compares the BER performances of our proposed CDD-OFDM scheme with $N_{T}=2,4$ transmit antennas with the single-antenna OFDM system under perfect and estimated channel information. It can be seen that our proposed MISO channel estimation scheme is only $1 \mathrm{~dB}$ away from the case of perfect channel knowledge and that the CDDOFDM system significantly outperforms the single-antenna OFDM system. For the 4-TX case, with $d=8$ and $r=1$,

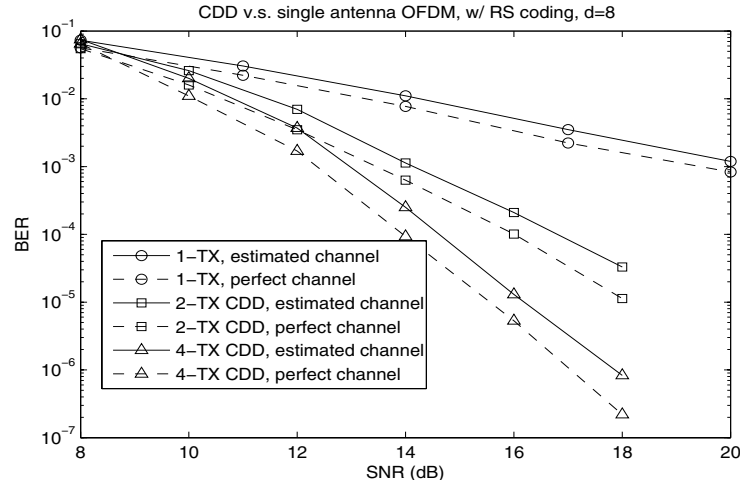

Fig. 6. BER comparison between CDD-OFDM and single-antenna OFDM with perfect and estimated channel knowledge

we set $\delta_{0}=2$ according to (26) and design the cyclic delay parameters according to (27). The cyclic delay parameters of the first OFDM symbol of the $n$-th antenna are set to $\Delta_{n}^{1}=64(n-1)$ where the factor of 64 is equal to the channel memory. It is clear that the slope of the BER curves at high SNR increases with $N_{T}$ due to the additional transmit diversity gain.

\section{CONCLUSION}

In this paper, we proposed a novel CDD scheme for pilotaided OFDM transmission which guarantees that the pilot codeword matrix is orthogonal to achieve the lowest channel estimation error variance for any number of transmit antennas without the need for full preamble OFDM symbols. Based on the original MISO channel model, our scheme estimates each channel individually and achieves significant performance improvement, at the same pilot overhead, compared to both SISO-equivalent and MISO channel estimation schemes for the conventional CDD-OFDM scheme which uses the same cyclic delay parameter for all OFDM symbols. Our simulation results for a DVB $2 \mathrm{~K}$ mode system show that the performance loss due to channel estimation is around $1 \mathrm{~dB}$.

\section{REFERENCES}

[1] G. Bauch and J. S. Malik, "Cyclic delay diversity with bit-interleaved coded modulation in orthogonal frequency division multiple access," IEEE Trans. Wireless Commun., vol. 5, pp. 2092-2100, Aug. 2006.

[2] S. M. Alamouti, "A simple transmit diversity technique for wireless communications," IEEE J. Select. Areas Commun., vol. 16, pp. 14511458, Oct. 1998

[3] V. Tarokh, H. Jafarkhani, and A. Calderbank, "Space-time block codes from orthogonal designs," IEEE Trans. Inform. Theory, pp. 1456-1467, July 1999.

[4] G. Auer, "Channel estimation for OFDM with cyclic delay diversity," in Proc. IEEE PIMRC, Sept. 2004.

[5] A. Dammann and S. Kaiser, "Standard conformable antenna diversity technique for OFDM and its application to DVB-T system," in Proc. IEEE GLOBECOM, Nov. 2001, pp. 3100-3105.

[6] S. M. Kay, Fundamentals of statistical signal processing: estimation theory. Upper Saddle River, NJ: Prentice Hall, 1993.

[7] T. Rappaport, Wireless Communications: Principles and Practice. Prentice Hall, 1996.

[8] ETSI EN 300744 V.1.5.1, "Digital Video Broadcasting (DVB): Framing structure, channel coding and modulation for digital terrestial television," ETSI, 2004.

[9] H. Oh, S. Kim, S.-H. Kim, and M.-G. Kim, "Novel transmit diversity techniques for broadcast services in cellular networks," in Proc. IEEE Veh. Techonol. Conf., vol. 2, May 2005, pp. 896 - 900. 